\title{
Folic acid exerts dose-dependent biphasic effects on cardiac de- velopment of zebrafish embryos
}

\author{
Xuhui Han 1,2,3, Bingqi Wang 1,2,3, Hongjie Wang 1,2,3 and Yao Zu 1,2,3,* \\ 1 International Research Center for Marine Biosciences, Ministry of Science and Technology, College of Fish- \\ eries and Life Sciences, Shanghai Ocean University, Shanghai, 201306, China. \\ 2 Key Laboratory of Exploration and Utilization of Aquatic Genetic Resources, Ministry of Education, College \\ of Fisheries and Life Sciences, Shanghai Ocean University, Shanghai, 201306, China. \\ 3 Key Laboratory of Freshwater Aquatic Genetic Resources, Ministry of Agriculture, College of Fisheries and \\ Life Sciences, Shanghai Ocean University, Shanghai, 201306, China. \\ * Correspondence: yzu@shou.edu.cn; Tel.: 86-21-61900405
}

Simple Summary: Folic acid is an essential vitamin for human beings. It has become a consensus to supplement folic acid during pregnancy. It is reported that $15 \% \sim 20 \%$ of people in the world supplement folic acid excessively. We found that excessive folic acid supplementation or insufficient folic acid intake in zebrafish could lead to abnormal heart development of zebrafish embryos. We elucidated the mechanism of folic acid on early cardiac development for the first time. These results provide a scientific basis for the important reasonable supplement of folic acid. At the same time, we constructed zebrafish mutants with abnormal folate metabolism, which provides a novel biological model for the study of folate metabolism.

\begin{abstract}
Folic acid, one of the 13 essential vitamins, plays an important role in cardiovascular development. Mutations in folic acid synthesis gene 5,10-methylenetetrahydrofolate reductase (MTHFR) is significantly associated with the occurrence of congenital heart disease. However, the mechanisms underlying the regulation of cardiac development by $m$ thfr gene are poorly understood. Here, we exposed zebrafish embryos to excessive folate or folate metabolism inhibitors. And we established a knock-out mutant of $m$ thfr gene in zebrafish by using CRISPR/Cas9. The zebrafish embryos of insufficient or excessive folic acid, and $m$ thfr-l- mutant all gave rise to early pericardial edema and cardiac defect at 3 days after fertilization(dpf). Furthermore, the folic acid treated embryos showed abnormal movement at $5 \mathrm{dpf}$. The expression levels of cardiac marker genes hand2, gata4 and nppa changed in the abnormality of folate metabolism embryos and $m t h \mathrm{fr}^{-/}$mutant, and there is evidence that they are related to the change of methylation level caused by the change of folate metabolism. In conclusion, our study provides a novel model for the in-depth study of MTHFR gene and folate metabolism. And our results reveal that folic acid has a dose-dependent biphasic effect on early cardiac development.
\end{abstract}

Keywords: $m$ thfr; folic acid; heart development; zebrafish; CRISPR/Cas9

Note: Authors are encouraged to provide a Graphical Abstract as a self-explanatory image to appear alongside with the text abstract in the Table of Contents. Figures should be a high-quality image in any common image format, and must be different from other figures in the main text. Note that images displayed online will be up to 11 by $9 \mathrm{~cm}$ on screen and the figure should be clear at this size. 


\section{Introduction}

Folic acid plays a vital role in cardiovascular development as it is an important vitamin necessary for methylation reaction, nucleotide synthesis and maintaining homocysteine at non-toxic level [1]. Insufficient folate metabolism will lead to methionine circulation obstruction and Hyperhomocysteinemia (Hhcy) [2]. Hhcy is an independent risk factor for congenital heart disease (CHD) [3]. Some studies have shown that folic acid deficiency can also affect development of brain, liver and other organs, leading to the occurrence of various diseases [4,5]. In the world, taking folic acid supplementation during pregnancy to prevent the occurrence of various congenital diseases has become a consensus. But there are still $15-20 \%$ of pregnant women due to the combined use of diet and folic acid supplements, resulting in 1-4 times the dose of excessive folic acid supplementation [6,7]. Therefore, it is very important to evaluate the effects of folic acid deficiency and excess on organ development.

Low folate intake during pregnancy and a common genetic variation in folate metabolism (methylenetetrahydrofolate reductase (MTHFR), 677C > t, about 15\% homozygous and $20 \%$ heterozygous in Caucasians, about $17 \%$ homozygous and $23 \%$ heterozygous in Northern Chinese) can lead to varying degrees of fetal dysplasia [8,9]. MTHFR is one of the key enzymes in folate pathway and methionine metabolism. Under the action of methylenetetrahydrofolate reductase and methionine synthetase reductase (MTRR), folate participates in two important aspects of metabolism in human body. Folic acid can add the chemical group of "one carbon unit" to the harmful homocysteine, thus reducing the level of Hcys in plasma [2]. It is very necessary to study the regulation of folic acid on early biological development.

The metabolism of folic acid includes the transformation of exogenous folic acid into 5-methyltetrahydrofolate (5-MTHF) and the methylation of 5-MTHF into Hcys [10,11]. As a carrier of one carbon unit, folic acid mediates the transfer of one carbon unit in the form of coenzyme in the process of amino acid metabolism and mutual transformation between methionine and Hcys [12,13]; These reports indicate that folic acid plays an important role in the early development of biological organs, especially in the development of neural tube. However, it is still unclear whether the excessive and deficient folate will affect the development of heart and the mechanism of folate affecting the development of organs including heart.

In this study, we used different concentrations of folic acid and folic acid inhibitor methotrexate to treat zebrafish embryos. We aim to explore the effects of excessive or insufficient folic acid supplementation on early heart development of zebrafish embryos. A zebrafish knock-out model of MTHFR gene was constructed by CRISPR/Cas9 technology to determine the dose base biphasic effect and mechanism of folic acid in early embryonic heart development. This study broadens our understanding of the potential function of MTHFR gene and deepens the current understanding of the relationship between folate and early heart development.

\section{Materials and Methods}

\subsection{Zebrafish maintenance and care}

The adult $\mathrm{AB}$ wild type zebrafish maintained at $28.5^{\circ} \mathrm{C}$ in the dark cycle of $14 \mathrm{~h} / 10 \mathrm{~h}$ light. Five to six pairs of zebrafish mate naturally. An average of 200-300 embryos were produced each time. Embryos were stored, washed and graded in E3 medium (5 mM $\mathrm{NaCl}, 0.17 \mathrm{~mm} \mathrm{KCl}, 0.33 \mathrm{~mm} \mathrm{CaCl}, 0.33 \mathrm{~mm} \mathrm{MgSO} 4$ dissolved in re distilled water) at $28.5 \mathrm{C}$. All handling of fishes was carried out in accordance with the guidelines on the care and use of animals for scientific purposes set up by the Institutional Animal Care and Use Committee (IACUC) of the Shanghai Ocean University (SHOU), Shanghai, China. This research was approved by the IACUC (IACUC 20171009) of SHOU.

\subsection{Microinjection of zebrafish embryos with CRISPR/Cas9 Knock-out}


The full-length codon-optimized Cas9 plasmid was obtained from Xiong' lab [14] sgRNAs were designed against the $m$ thfr gene (ENSDARG00000053087) using the CRISPR design tool (http://crispr. mit.edu/) [15]. The target was designed as Exon2 5' - GGTGAACCAAAGAGCTGACG - $3^{\prime}$. The sequence of primers was as follows Primers forward 5'GGGGTAATGCTGCCAACTGA - $3^{\prime}$ Primers reverse 5'- GATTGACCGCTCCAGACGAT $-3^{\prime}$. Using protocol of message machine ${ }^{\circledR}$ T7 ultra Kit (Thermo Fisher Scientific, USA, AMB13455) was transcribed in vitro to synthesize cas9 mRNA, and maxiscriptt7 was used to transcribe in vitro, and then $\mathrm{sgRNA}$ was obtained by $\mathrm{LiCl} /$ ethanol precipitation. At the single cell stage, embryos were injected with $1 \mathrm{nl}$ solution containing $80 \mathrm{ng} / \mu \mathrm{l}$ thfr-sgRNAs and 400ng/ $\mu \mathrm{l}$ cas9 protein. Then the injected embryos were cultured in E3 medium.

\subsection{In situ hybridization}

Whole mount in situ hybridization (WISH) was performed as previously described by $\mathrm{Zu}$ et al [16]. Primers used for antisense probe synthesis were listed in supplementary Table S1.

\subsection{Quantitative Real-time PCR}

Total RNA was extracted by homogenizing 30 embryos in Trizol reagent (Invitrogen, USA,15596-026), followed by standard reverse transcription. Using SYBR ${ }^{\circledR}$ Green Master Mix (Thermo Fisher, USA, A25742) was used for quadruplicate real-time PCR. Primer sequences are listed in the supplementary table $\mathrm{S} 2 . \beta$-actin was used to standardize the gene level. The change of multiple was calculated by $2^{-\Delta \Delta}$ method. Statistically significant difference was defined as the $\mathrm{P}<0.05$ threshold of student's $\mathrm{T}$ test.

\subsection{Behavioral test}

Behavioral tests were performed at $5 \mathrm{dpf}$ and recorded using Danio vision system (Noldus information technology company, Wageningen, the Netherlands). Zebrafish were divided into wild type, MTX antagonistic and folic acid excess group. Embryos were collected and placed in a 24 well E3 medium. After 15 minutes of indoor adaptation, 10 minutes of exercise were recorded. Digital tracks and heat maps were generated using the Ethovision ${ }^{\circledast}$ XT 11.5 software (Noldus). The above measurements are made in triplicate.

\subsection{Assessment of DNA methylation level}

Bisulfite sequencing PCR (BSP) was used to verify the change of DNA methylation level. Genomic DNA was treated with bisulfite according to the operation manual of EZ DNA methylation gold ${ }^{\circledR}$ Kit (Zymo, USA, d5005). The primers of bsp-pcr were designed by methprimer (http://www.urogene.org/cgi-bin/methprimer/ methprimer.cgi). The primer sequence is in the supplementary table. DNA fragments were cloned and sequenced by trans1-t1 competent cells. BIQ analyzer software was used to analyze the sequencing results.

\section{Results}

3.1. Folic acid has biphasic effects on early heart development in zebrafish

We evaluated cardiac development in transgenic zebrafish $\operatorname{Tg}(\mathrm{cmlc2}$ :EGFP ; $\mathrm{krdl}: \mathrm{m}$ Cherry) embryos using fluorescent labeling to investigate the role of exogenous folate in cardiac development after normal neural tube closure (Fig. 1A). Embryos were 


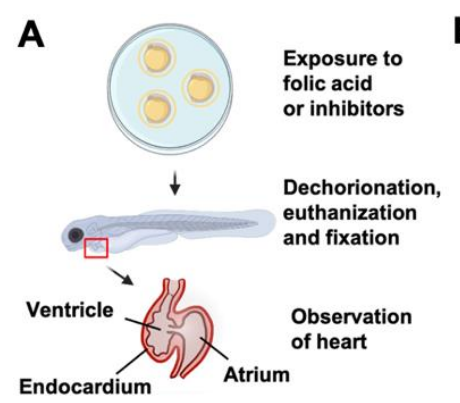

B C

$\mathbf{E}$
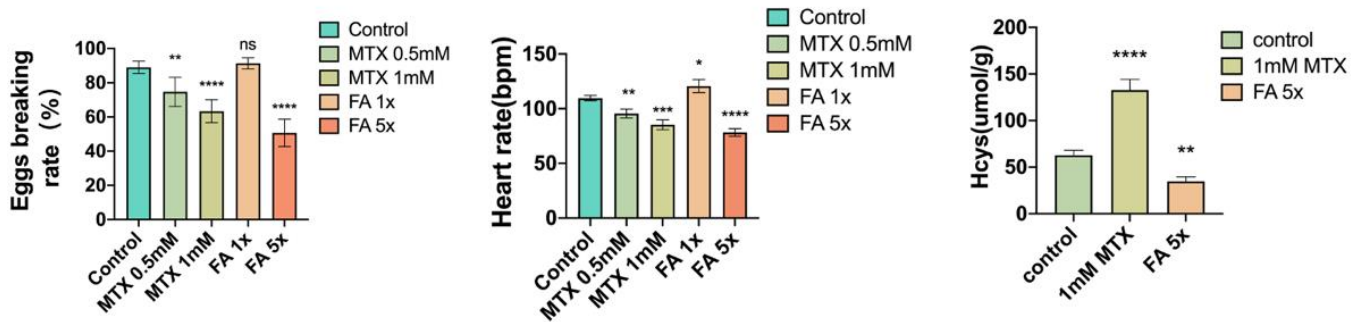

D

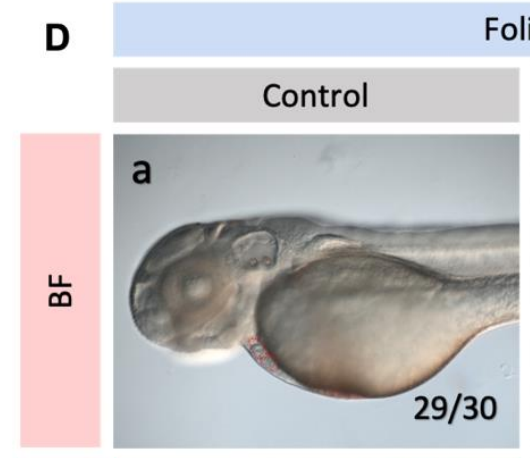

Folic acid excess or antagonistic treatment of cardiac phenotypes
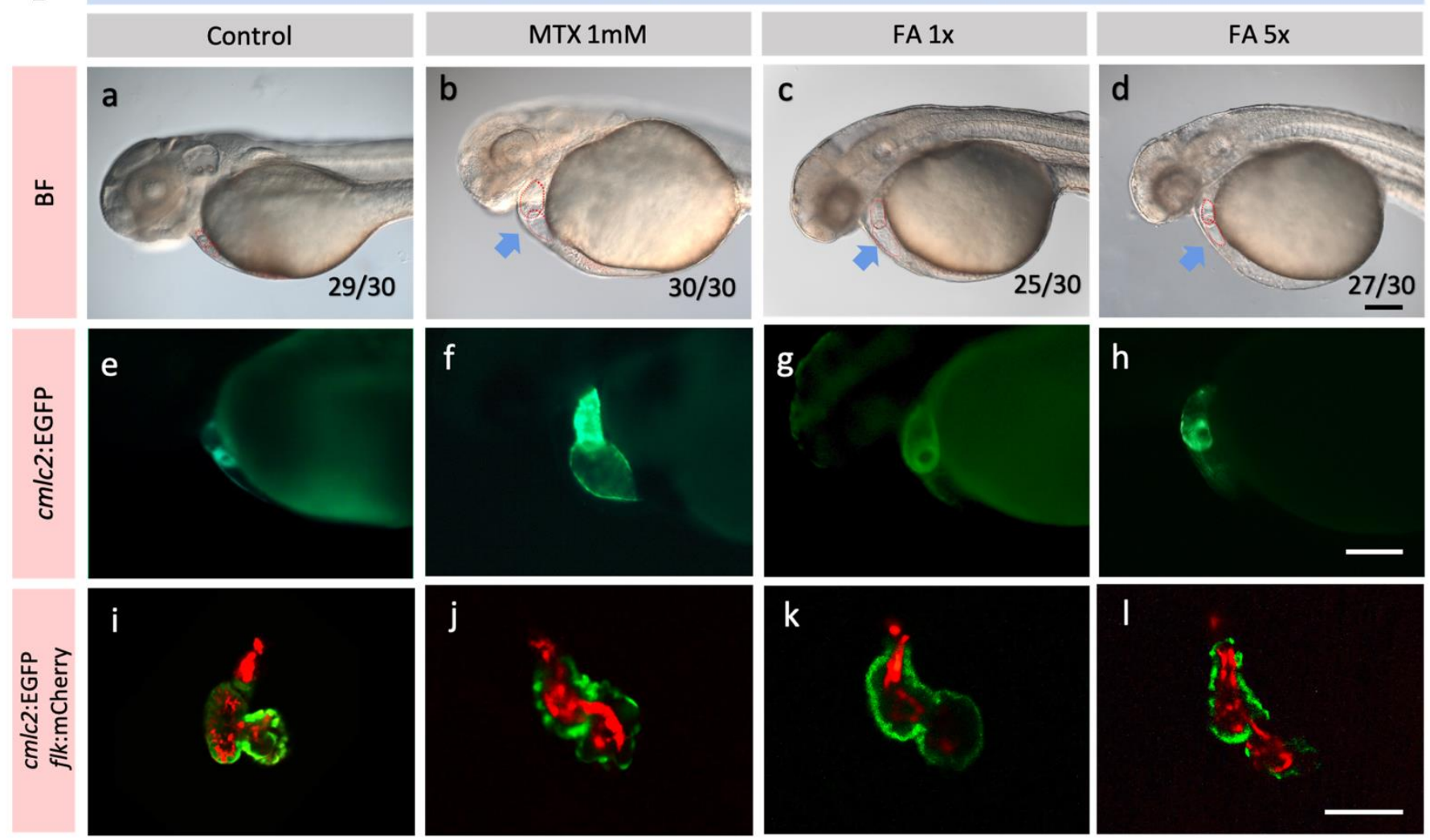

$\mathbf{F}$
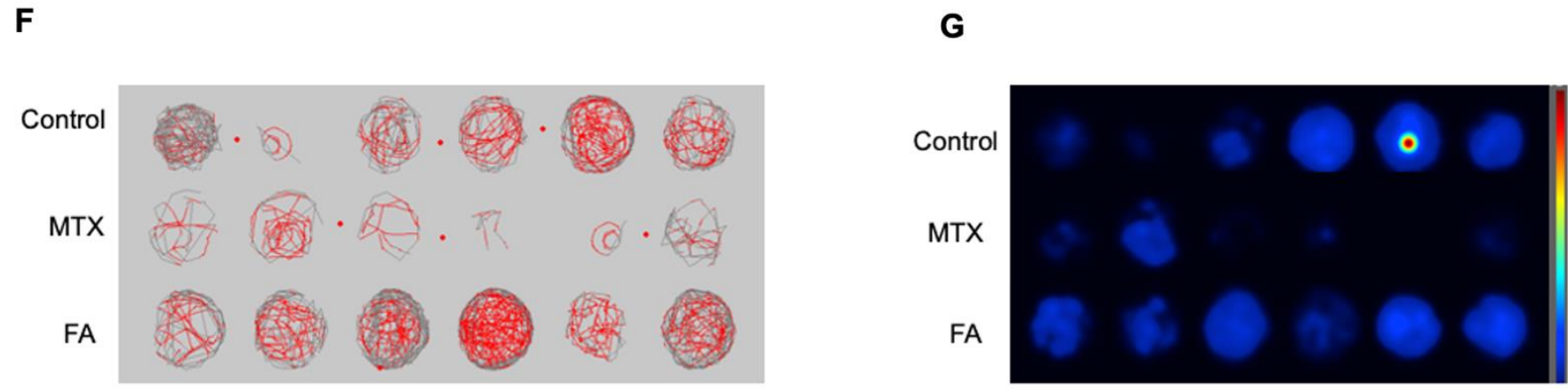

Figure 1. Folate excess and folate deficiency can lead to different degrees of abnormal development of cardiac physiological function and abnormal metabolism of homocysteine. (A) Treatment of zebrafish embryos with folic acid and folic acid inhibitors. (B) The rate of membrane rupture of zebrafish embryos treated with folic acid and folic acid inhibitor was statistically analyzed. $\left({ }^{*} \mathrm{p}<0.05,{ }^{* *} \mathrm{p}<0.01,{ }^{* * *} \mathrm{p}<0.001,{ }^{* * * *} \mathrm{p}<0.0001\right.$, ns: no statistical difference) (C) The rate of membrane rupture of zebrafish embryos treated with folic acid and folic acid inhibitor was statistically analyzed. (D) The phenotypes of zebrafish 3dpf embryonic heart after different concentrations of folic acid and MTX inhibited folate metabolism pathway were demonstrated. a-d is the view under light microscope, e-h is cmlc2:EGFP labeled cardiac fluorescence image, $\mathrm{i}-\mathrm{l}$ is cmlc2:EGFP and flk:mCherry Co labeled confocal images of the heart. Scale bars: $100 \mu \mathrm{m}$. (E) High homocysteine content 
in zebrafish of different groups. (F) Digital tracks of larvae from wildtype (WT), MTX and FA 1x groups at 5 dpf. (G) Heat maps of the digital tracks F.

kept in E3 medium and exposed to inhibitors methotrexate (MTX) and different doses of folic acid. Therefore, the exposure time was $16 \mathrm{hpf}$ to $36 \mathrm{hpf}$. We set up five groups of exposure, namely control group, $0.5 \mathrm{mM}$ and $1 \mathrm{mM} \mathrm{MTX}$, recommended [7] dose of folic acid (FA $1 \mathrm{x}, 1 \mathrm{mM})$ and five times excess dose of folic acid (FA 5x,5mM). There were 30 embryos in each group, and three biological repetitions were set. At the end of exposure, the $36 \mathrm{hpf}$ eggs breaking rate of zebrafish embryos was counted. With the increase of antagonist and folic acid dosage, the normal membrane breaking rate of zebrafish embryos decreased (Fig. 1B). The heart rate of zebrafish embryos decreased with the use of antagonists and excessive folic acid (Fig. 1C). These results show that excessive folic acid supplementation and use of antagonists can lead to delayed embryonic development of zebrafish.

The cardiac development of zebrafish was observed at $36 \mathrm{hpf}$. The results showed that folic acid inhibitor could make the pericardial cavity of zebrafish embryos swell and the ventricles expand in different degrees. Excessive folic acid can make zebrafish embryo atria and ventricles elongate and heart looping abnormal (Fig. 1D f,g,h). These results suggest that both folic acid deficiency and folic acid excess can lead to abnormal cardiac development in zebrafish embryos, which is usually positively correlated with the dose. Confocal images showed that zebrafish heart looping was abnormal after different gradients of folic acid and MTX folic acid antagonist treatment. At the same time, the structure of cardiac chamber also changed, and the thickness of myocardial wall also changed more and more obviously with the change of treatment dose (Fig. 1D j,k,i).The cardiac development of zebrafish was observed at $36 \mathrm{hpf}$. The results showed that folic acid inhibitor could make the pericardial cavity of zebrafish embryos swell and the ventricles expand in different degrees. Excessive folic acid can make zebrafish embryo atria and ventricles elongate and heart looping abnormal (Fig. 1D f,g,h). These results suggest that both folic acid deficiency and folic acid excess can lead to abnormal cardiac development in zebrafish embryos, which is usually positively correlated with the dose. Confocal images showed that zebrafish heart looping was abnormal after different gradients of folic acid and MTX folic acid antagonist treatment. At the same time, the structure of cardiac chamber also changed, and the thickness of myocardial wall also changed more and more obviously with the change of treatment dose (Fig. 1D j,k,i).

3.2. Deficiency of folic acid metabolism in zebrafish embryos leads to significant increase of Hcys content

The content of Hcys in zebrafish embryos of different treatment groups was determined. The results showed that insufficient folate metabolism would lead to significant increase of Hcys content. However, excessive folic acid supplementation can keep Hcys at a low level (Fig. 1E).

We observed abnormal behavior changes in zebrafish treated with folic acid or $m t h f r$ mutant. behavior tests are also carried out at $5 \mathrm{dpf}$. The digital trajectory and the corresponding heat map are shown in figures $1 \mathrm{~F}$ and $\mathrm{G}$ (Fig. 1F-G). Compared with wild-type embryos, the movement distance and average velocity of $m t h f r-$ mutant embryos were observed (Fig. S1). Mobility is the percentage of the completed area (Fig. S1 C). Zebrafish showed decreased movement state are similar to human Hyperhomocysteinemia. Different concentrations of folate were used in HEK293T cells and detected by luciferase reporter gene system. We found that the change of folate content can also cause the change of some genes related to cardiac development regulation (Fig. S2). 
A

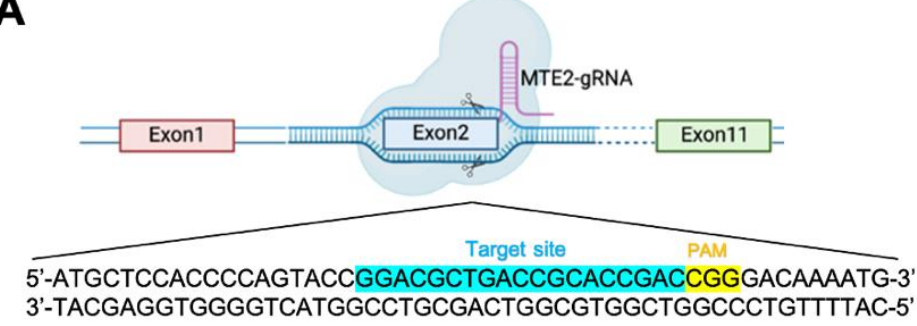

mthfr CRISPR-induced mutations in F1 WT bacground

WT GGACGCTGACCGCACCGACCGGGAC

F1 GGACGCTGACCGC-----GACCGGGAC $\triangle 3$

F1 GGACGCTGA-_-ACCGGGAC $\triangle 8$

F1 GGACGCTGAC-1-ACCGGGAC $\triangle 7$

C
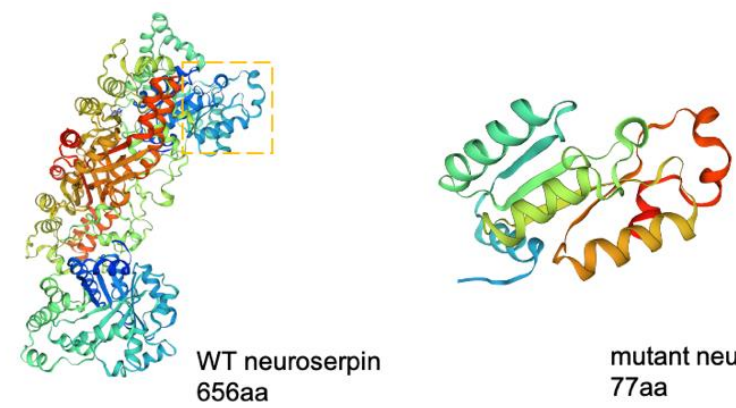

mutant neuroserpin

$77 a a$

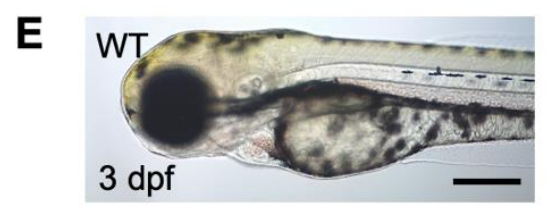

B

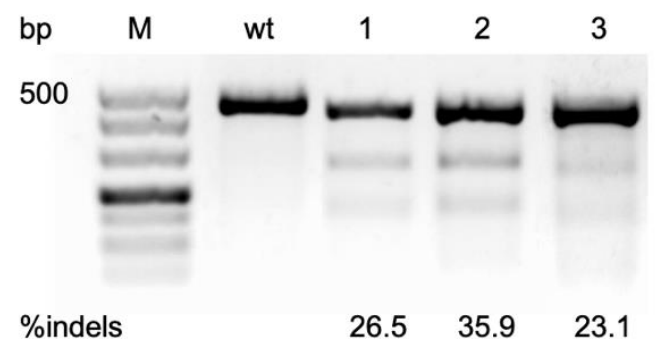

D

GGACGCTGACCGCACCGACCGGGACAAAATGA

WT

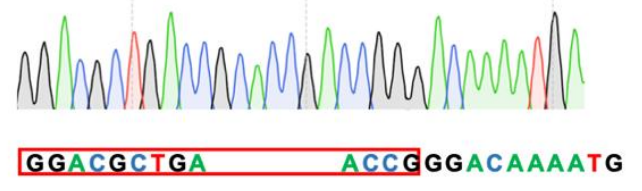

mthfr-/-

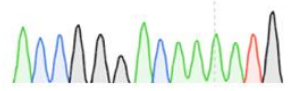

$\mathbf{F}$

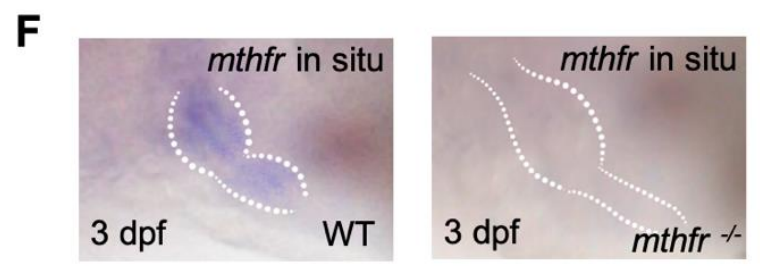

Figure 2. Generation of $m$ thfr mutant using the CRISPR/Cas9 system. (A) Design of sgRNA target in exon 2 of zebrafish mthfr gene. The mutation type of code shift selected by orange frame is selected finally. (B) Two short DNA fragments were obtained by T7E1 digestion. In the control group, $437 \mathrm{bp}$ fragment was amplified from wild-type embryos with the same set of primers. (C) The predicted truncation of Mthfr protein. (D) Sequencing map of homozygous zebrafish adults. (E) Bright-field views of $m$ th fr-l- $^{-1}$ zebrafish showed Pericardial enlargement, ventricular enlargement and abnormal heart looping. Scale bars: $500 \mu \mathrm{m}$. (F) The results of in situ hybridization showed that the expression of $m$ thfr gene in homozygous mutant zebrafish was changed.

\subsection{CRISPR/Cas9 mediated mthfr gene knock-out model in zebrafish}

We constructed the mthfr gene knock-out mutant by using CRISPR/Cas9 in zebrafish. mthfr gene was knocked out by a specific sgRNA target on exon 2 (Fig. 2A). Microinjection of $m t h f r$ sgRNA binding Cas 9 protein into the fertilized single-cell embryos was performed. RT-PCR was performed 2 days after fertilization to verify the indels of exon 2 of $m$ thfr transcript in the injected embryos. The results showed that the injection of $m$ thfr knock-out gRNA resulted in the indels of exon 2. One fragment was amplified with primers spanning the exon 2 gRNA sequence of $m t h f r$, and two shorter DNA fragments were obtained by T7E1 digestion. In the control group, a $437 \mathrm{bp}$ fragment was amplified from wild-type embryos (Fig. 2B). Sequencing of PCR products confirmed that the exon 2 of $m$ thfr knock-out injected embryo transcript had several base pairs deletion. The genome editing efficiency of several mthfr sgRNA targeting different sites was compared, and the one with the highest efficiency for exon 2 was selected. It is predicted that the $8 b p$ frameshift deletion will produce premature stop codon, which will truncate the protein of 77 amino acids (aa) compared with 656aa full-length Mthfr protein (Fig. 2C). The mthfr fish carrying $8 \mathrm{bp}$ deletion was hybridized to obtain homozygous $m$ thfr fish whose genotype was determined by sequencing (Fig. 2D). Compared with wild-type zebrafish embryos, $m$ thfr $\%$ mutant zebrafish embryos have obvious abnormal cardiac development. 
Pericardial enlargement, ventricular enlargement and abnormal heart looping were found (Fig. 2E).

In situ hybridization showed that $m$ thfr gene was expressed at $3 \mathrm{dpf}$ wild-type zebrafish heart. The expression of $m$ thfr gene decreased significantly in $m t h f r-1$ mutant. (Fig. 2F-G). Real qPCR experiment showed that the expression of $m$ thfr gene increased at 6hpf, then decreased and stabilized at $12 \mathrm{hpf}$ (Fig. S3 A). In situ hybridization confirmed that $m$ thfr was widely expressed in zebrafish embryos, including heart, brain, optic nerve and liver. It is strongly expressed in the heart (Fig. S3 B). These results indicate that $m$ thfr gene is involved in folate metabolism and plays an important role in early embryonic development of zebrafish. After 16hpf neural tube closure [17], mthfr gene is still involved in the later organ development.

A Some heart development related genes
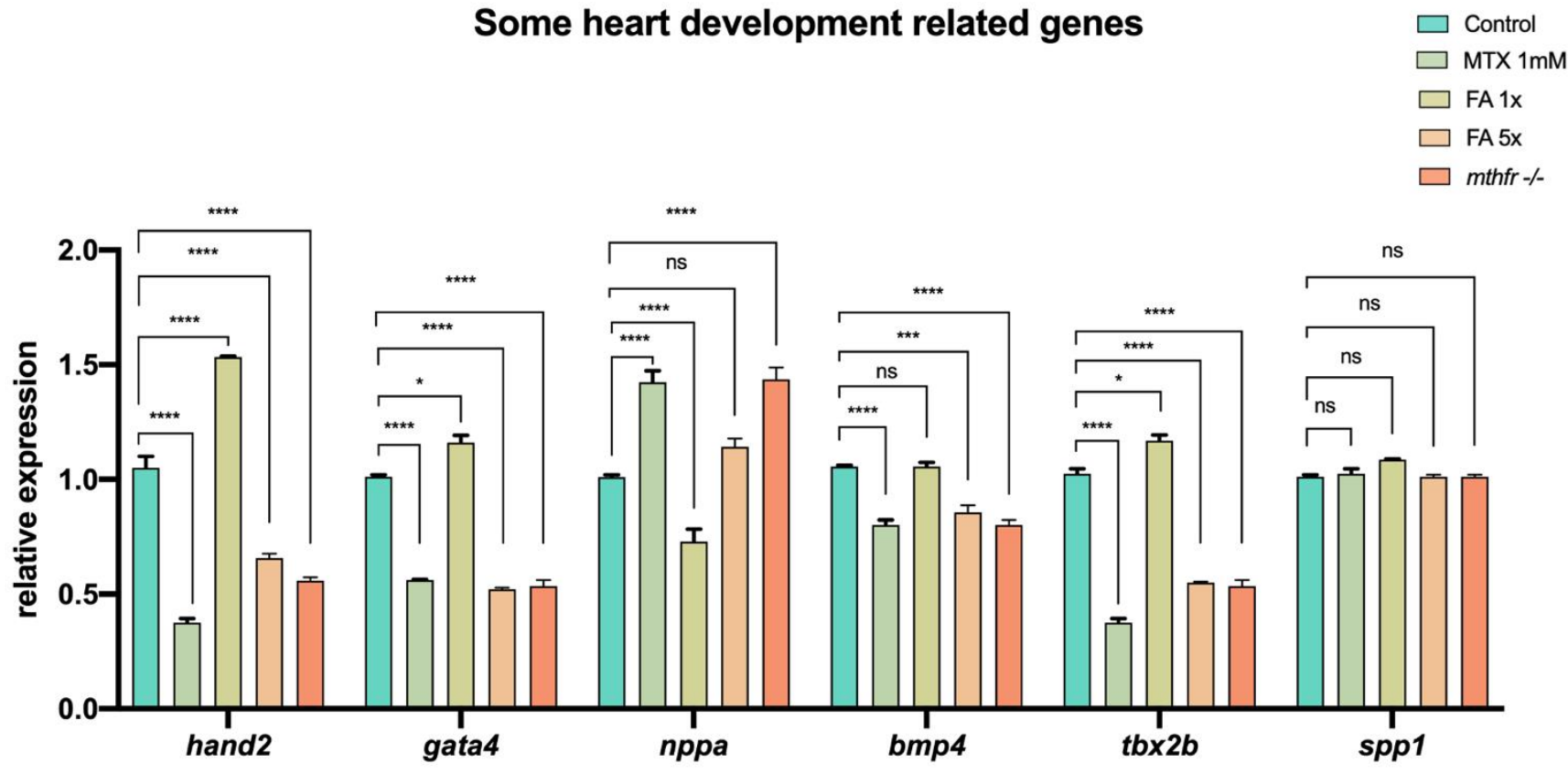

B

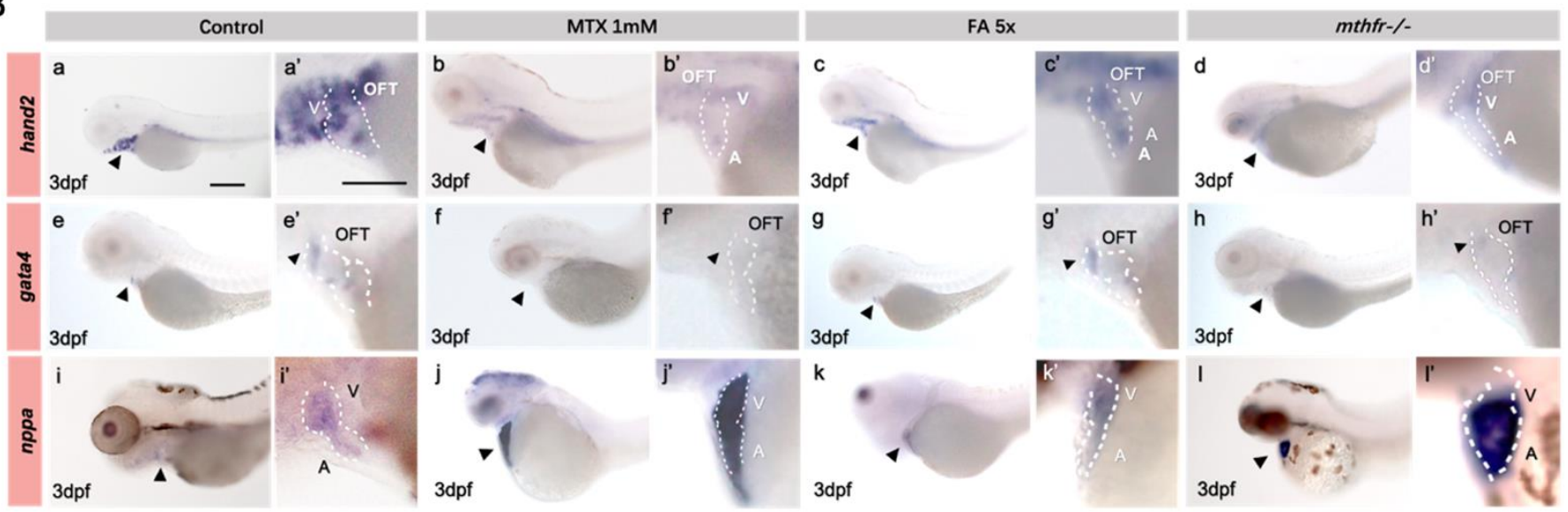

Figure 3. Abnormal folate metabolism results in changes of zebrafish embryo heart development gene expression. (A) Results of Q-PCR for hand2, nkx2.5, gata4, bmp4, tbx2b, spp1, hoxb1a, nppa in the embryonic heart of zebrafish in each group. (Student -t test, ${ }^{*} \mathrm{p}<0.05,{ }^{* *} \mathrm{p}<0.01,{ }^{* * *} \mathrm{p}<0.001,{ }^{* * * *} \mathrm{p}<0.0001$, ns: no statistical difference) .(B) In situ hybridization showed the expression of hand2, gata4, nppa in different groups of 3dpf zebrafish embryonic heart. A: atria V: ventricle OFT: outflow tract. $n=3$. Scale bars: $200 \mu \mathrm{m}$.

3.4. Folic acid deficiency and excessive supplement lead to the changes of heart related genes. 
The expressions of hand2, gata4, nppa, bmp4, tbx2b, spp1 in the heart of zebrafish embryos at 3dpf were detected semi quantitatively by Q-PCR. Compared with the untreated control group, the changes of folate supplement and the expression of genes related to heart development in mthfr ${ }^{-/-}$mutant embryos were different (Fig. 3A).

According to the results of Q-PCR and the previous abnormal phenotype of heart, selected hand2, gata4 and nppa as the key genes of heart development and indicators for in situ hybridization. In wild-type zebrafish embryos, hand2 was expressed in the AVC(A-V canal) of the heart at $3 \mathrm{dpf}$ (Fig. 3Ba). The expression of hand2 in zebrafish embryonic heart was decreased in folic acid antagonistic treatment group, folic acid excess supplement group and $m t h f r-$ mutation group (Fig. 3Bb-d). In wild-type zebrafish embryos, gata4 was strongly expressed in cardiac outflow tract (OFT) and AVC (Fig. 3Be). Similarly, the expression of gata4 gene decreased with the metabolism of folic acid (Fig. 3Bf-h). This is consistent with the Q-PCR expression trend of hand2. In zebrafish embryonic heart, nppa gene expression was up-regulated with the decrease of folate metabolism (Fig. 3Bi-1).

A

BSP-hand2

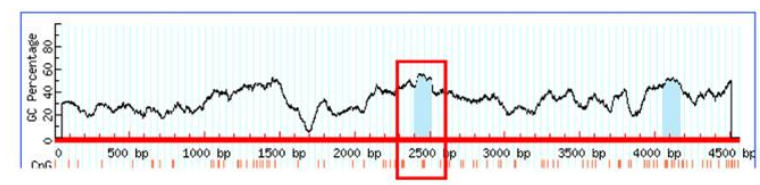

B

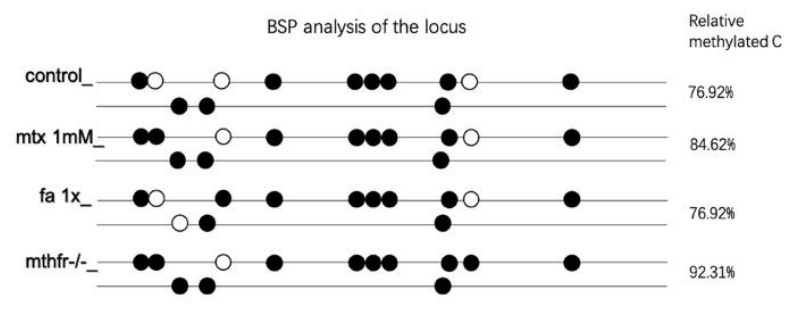

$\mathrm{E}$

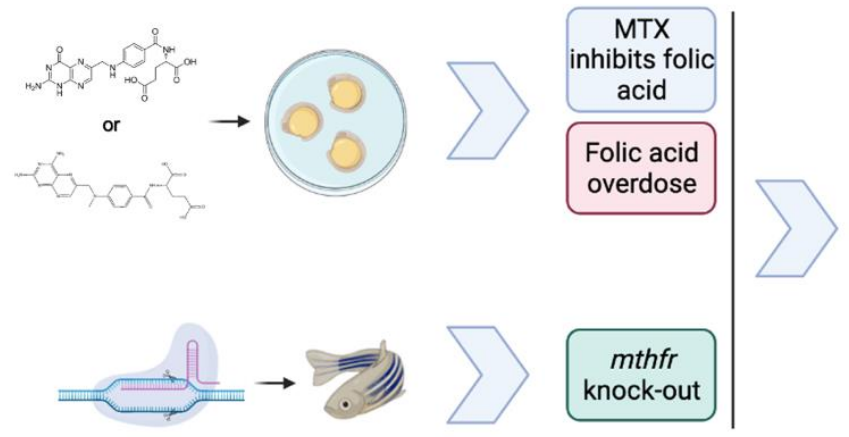

C
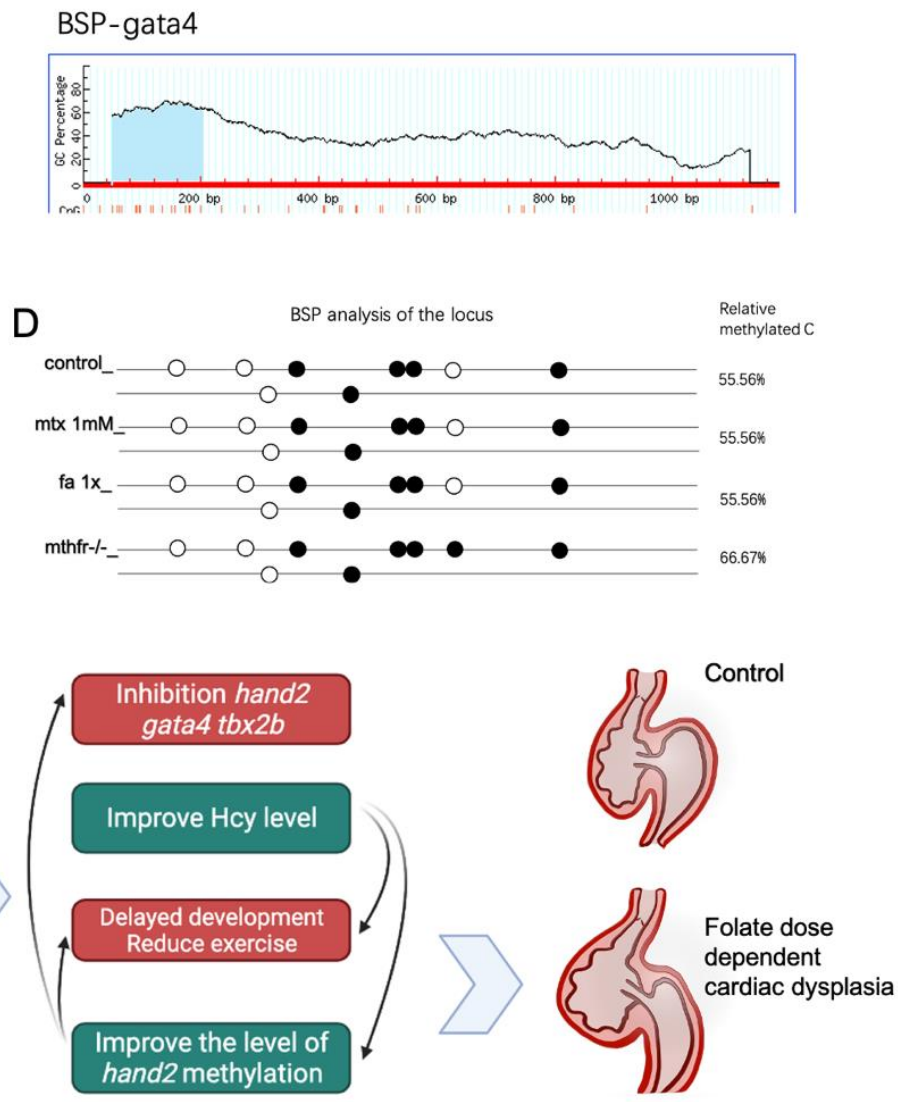

Figure 4. Effects of folic acid on partial gene methylation in zebrafish embryos. (A) The CpG island of hand2 gene for BSP experiment was predicted and selected. (B) Zebrafish embryos with different folate metabolism groups BSP analysis of the locus of hand2. (C) The CpG island of gata4 gene for BSP experiment was predicted and selected. (D) Zebrafish embryos with different folate metabolism groups BSP analysis of the locus of gata4. (E) The schematic diagram of the dose-dependent biphasic effects mechanism of folic acid on zebrafish heart development.

\subsection{The abnormal methylation of hand2 and gata4 were caused by folate metabolism changes}

Epigenetic regulation of gene expression involves many processes[18]. We used bisulfite sequencing (BSP) to verify that abnormal folate metabolism can change the methylation level of hand2 gene. BSP primer sequence was designed by predicting the CpG island of hand2 gene promoter (Fig. 4A). The results showed that folate inhibition and 
abnormal folate metabolism caused by mthfr -/- mutation could increase the methylation level of hand2 gene (Fig. 4B). The increase of promoter methylation level can inhibit the expression of hand 2 in zebrafish early embryonic development. Compared with the effect of folic acid on the methylation level of hand2 gene, the change of folic acid content had no significant effect on the methylation level of gata4 gene. Only in mthfr -/ mutant, the methylation level of gata4 gene was slightly up-regulated (Fig. 4D). Based on the existing results, the effect of folic acid dose-dependent two-way effect on zebrafish heart development is shown in Figure 4E.

\section{Discussion}

This study found that excessive or insufficient folate can lead to abnormal heart development, and $m$ thfr gene is one of the key genes. We selected $16 \mathrm{hpf}$ as the initial period of folic acid or MTX exposure. At about $16 \mathrm{hpf}$, the neural tube of zebrafish closed normally [19]. At $24 \mathrm{hpf}$, the original cardiac tube was formed by the differentiation of cardiac cells. At $36 \mathrm{hpf}$, the differentiation of atria and ventricles was basically completed, and cardiac morphogenesis and torsion completed the development of cardiac looping [20,21] . Although it has been reported that MTHFR gene is closely related to folate metabolism, DNA synthesis and methylation [22], and it has been recognized that folic acid supplementation during pregnancy is beneficial to fetal development, but the mechanism of MTHFR gene mutation leading to cardiac dysplasia and increased risk of congenital heart disease is still unclear. We have constructed a zebrafish strain with mthfr knock-out, which provides a novel model for exploring the function of $m$ thfr in the development of various organs, folate metabolism and DNA methylation of zebrafish early embryos.

Folic acid deficiency could lead to the increase of Hcys. Interestingly, excessive folate supplementation also led to similar phenotypes and gene expression changes in zebrafish embryos as folate deficiency. Previous studies have shown that folic acid is converted into 5-methyltetrahydrofolate (5-MTHF) in vivo and 5-MTHF enters the methylation reaction of Hcys [23]. Hhcy is an independent risk factor for cardiovascular disease. Hhcy can lead to fatigue, emotional changes and reduced exercise status [24]. However, excessive folic acid did not increase Hcys. These results suggest that folic acid supplementation can lead to changes in the expression of genes related to cardiac development, but it does not affect the cycle of one carbon unit and the transformation process of folic acid to active folic acid. Studies have shown that the combined effect of genetic and environmental factors may be the main cause of congenital heart disease [21]. The development of heart is a complex process, many factors participate in the regulation of heart development. The disorder of these factors can cause the abnormal development of the heart. hand2, gata4, nppa, bmp4, $t b x 2 b$, spp 1 genes expressed in Second heart field, cardiac chamber and atrioventricular septum are very important for early cardiac development [25]. In this study, the expression of hand2, gata4, bmp4, tbx2b, nppa showed a dose-dependent change of folic acid. Except $n p p a$, the change trend of these gene expression is consistent. In addition, excessive folate treatment and folate inhibitor treatment led to down-regulation of gene expression. The results of these in situ hybridization experiments were consistent with the previous results of Q-PCR. This proves that folate excess and deficiency have a biphasic dose-dependent relationship with some genes related to heart development and folate metabolism.

In addition, from the previous Hcys results and behavioral experiments, we found that the increase of exogenous folate could make zebrafish more active. Q-PCR results and in situ hybridization data revealed that the expression of $m t h f r$ gene, hand 2 and other cardiac development related genes were more stable and balanced due to the normalization of folate cycle. The homocysteine level in zebrafish tends to be normal. Therefore, the heart development of zebrafish embryos tends to be normal. This also explains why abnormal folate metabolism leads to decreased heart rate and increased signs of heart failure. 
As S-adenosylmethionine (SAM) is the most common methyl donor of DNA methylation, it can directly affect the degree of DNA and protein methylation, thus causing apparent genetic disorder [26]. Folic acid acts as the carrier of one carbon unit in the process of amino acid metabolism and the mutual transformation of methionine and Hcys and mediates the transfer of one carbon unit in the form of coenzyme. SAM involved in folate cycle, will also be generated during folate cycle. In this study, we determined that folic acid deficiency could increase the methylation level of hand2 gene promoter. The addition of folic acid can keep DNA methylation at a stable level.

Overall, we constructed a novel mthfr zebrafish mutation model and found the first time that insufficient or excessive folic acid intake can lead to changes in gene expression during early cardiac development of zebrafish embryos. Abnormal folate metabolism can lead to abnormal metabolism of Hcys and abnormal methylation of some genes in zebrafish. These results will increase the risk of all kinds of congenital diseases, including congenital heart disease. It has been proved that excessive folic acid supplementation has a negative effect on the early development of organisms. Folic acid has a dose-dependent biphasic effect on organism development.

\section{Conclusions}

Folic acid has biphasic effects on early heart development in zebrafish.The differential expression of cardiac development related genes is related to folate.Abnormal folate metabolism changes the methylation level of hand2 and gata4 promoters.

Supplementary Materials: The following are available online at www.mdpi.com/xxx/s1, Figure S1: Behavioral data analysis in zebrafish embryos caused by abnormal folate metabolism, Figure S2:Different concentrations of folate were used in HEK293T cells and detected by luciferase reporter gene system, Figure S2: $m$ thfr gene is widely expressed in zebrafish embryos and qPCR data at different developmental stages, Table S1: Primers used for antisense probe synthesis, Table S2: Primers that were used for Quantitative Real-time Polymerase Chain Reaction Video S1: mthfr homozygous mutant zebrafish phenotype video.

Author Contributions: Conceptualization, Y.Z., X.H.; methodology, X.H.; software, X.H.; validation, X.H.and B.W.; formal analysis, X.H.; in-vestigation, X.H.; resources, X.H.; data curation, X.H. and H.W.; writing-original draft preparation, X.H and Y.Z..; writing-review and editing, X.H., Y.Z. and H.W.; visualization, X.H.; supervision, Y.Z.; project administration, X.H. and Y.Z.; funding acquisition, Y.Z. All authors have read and agreed to the published version of the manuscript.

Funding: The work is supported by grants from the National Key Research and Development Program of China 2018YFD0900601, National Natural Science Foundation of China 32170423 and 31501166, Chenguang Program 14CG49、Major Science Innovation Grant 2017-01-07-00-10-E00060 from the Shanghai Education Committee, and Shanghai Sailing Program of Science and Technology Commission of Shanghai Municipality 15YF1405000.

Institutional Review Board Statement: All handling of fishes was carried out in accordance with the guidelines on the care and use of animals for scientific purposes set up by the Institutional Animal Care and Use Committee (IACUC) of the Shanghai Ocean University (SHOU), Shanghai, China. This research was approved by the IACUC (IACUC 20171009) of SHOU.

Informed Consent Statement: Not applicable.

Data Availability Statement: All data needed to evaluate the conclusions in the paper are included in the paper and/or the Supplementary Materials. The plasmid system and fish lines used in this study are available from the corresponding author upon reasonable request.

Acknowledgments: We thank Dr. Jingwei Xiong (Institute of Molecular Medicine, Peking University) for providing the pUC19-scaffold plasmid for gRNA synthesis. We thank Wenhao Li for data analysis assistance.

Conflicts of Interest: The authors declare no conflict of interest. 
1. P. Verhoef, M.J. Stampfer, Prospective Studies of Homocysteine and Cardiovascular-Disease, Nutrition Reviews 53 (1995) $283-$ 288.

2. L. Bethke, E. Webb, et al., Functional polymorphisms in folate metabolism genes influence the risk of meningioma and glioma, Cancer Epidemiol Biomarkers Prev 17 (2008) 1195-1202.

3. T. Deloughery, Common mutation in methylenetetrahydrofolate reductase. Correlation with homocysteine metabolism and late-onset vascular disease, Circulation 94 (1996) 3074.

4. N.L. Morse, Benefits of docosahexaenoic acid, folic acid, vitamin D and iodine on foetal and infant brain development and function following maternal supplementation during pregnancy and lactation, Nutrients 4 (2012) 799-840.

5. K.E. Christensen, L.G. Mikael, et al., High folic acid consumption leads to pseudo-MTHFR deficiency, altered lipid metabolism, and liver injury in mice, Am J Clin Nutr 101 (2015) 646-658.

6. L. Plumptre, S.P. Masih, et al., High concentrations of folate and unmetabolized folic acid in a cohort of pregnant Canadian women and umbilical cord blood, Am J Clin Nutr 102 (2015) 848-857.

7. L.K. Murray, M.J. Smith, N.M. Jadavji, Maternal oversupplementation with folic acid and its impact on neurodevelopment of offspring, Nutr Rev 76 (2018) 708-721.

8. A.I. Scher, G.M. Terwindt, et al., Migraine and MTHFR C677T genotype in a population-based sample, Ann Neurol 59 (2006) 372-375.

9. B. Yang, S. Fan, et al., Associations of MTHFR C677T and MTRR A66G gene polymorphisms with metabolic syndrome: a casecontrol study in Northern China, Int J Mol Sci 15 (2014) 21687-21702.

10. J. Fan, J. Ye, et al., Quantitative flux analysis reveals folate-dependent NADPH production, Nature 510 (2014) 298 -302.

11. C.A. Hobbs, M.A. Cleves, et al., National Birth Defects Prevention, Conotruncal heart defects and common variants in maternal and fetal genes in folate, homocysteine, and transsulfuration pathways, Birth Defects Res A Clin Mol Teratol 100 (2014) 116126.

12. D.A. Wang, F. Wang, et al., Lower Circulating Folate Induced by a Fidgetin Intronic Variant Is Associated With Reduced Congenital Heart Disease Susceptibility, Circulation 135 (2017) 1733.

13. S. Saini, V. Sharma, et al., 170 Effect of Folic Acid Supplementation on In Vitro Maturation of Oocytes and Folate Cycle, Reproduction Fertility and Development 30 (2018) 224.

14. D. Liu, Z.X. Wang, et al., Efficient Gene Targeting in Zebrafish Mediated by a Zebrafish-Codon-Optimized Cas9 and Evaluation of Off-Targeting Effect, J Genet Genomics 41 (2014) 43-46.

15. Y.H. Zhang, W. Qin, et al., Programmable base editing of zebrafish genome using a modified CRISPR-Cas9 system, Nat Commun 8 (2017).

16. X.J. Tong, Y. Zu, et al., Kctd10 regulates heart morphogenesis by repressing the transcriptional activity of Tbx5a in zebrafish, Nat Commun 5 (2014).

17. A. Aquilina-Beck, K. Ilagan, Q. Liu, J.O. Liang, Nodal signaling is required for closure of the anterior neural tube in zebrafish, Bmc Dev Biol 7 (2007).

18. P.A. Jones, Functions of DNA methylation: islands, start sites, gene bodies and beyond, Nat Rev Genet 13 (2012) $484-492$.

19. L.A. Lowery, H. Sive, Strategies of vertebrate neurulation and a re-evaluation of teleost neural tube formation, Mech Develop 121 (2004) 1189-1197.

20. P. Polar, M.T.K. Kairo, et al., Assessment of fungal isolates for development of a myco-acaricide for cattle tick control, VectorBorne Zoonot 5 (2005) 276-284.

21. J. Le Notre, L. Brissieux, et al., Tandem isomerization/Claisen transformation of allyl homoallyl and diallyl ethers into gamma,delta-unsaturated aldehydes with a new three component catalyst Ru-3(CO)(12)/imidazolinium salt/Cs2CO3, Chem Commun (2002) 1772-1773.

22. H.J. Sofia, G. Chen, B.G. Hetzler, J.F. Reyes-Spindola, N.E. Miller, Radical SAM, a novel protein superfamily linking unresolved steps in familiar biosynthetic pathways with radical mechanisms: functional characterization using new analysis and information visualization methods, Nucleic Acids Res 29 (2001) 1097-1106.

23. K.J.A. Lievers, G.H.J. Boers, et al., A second common variant in the methylenetetrahydrofolate reductase (MTHFR) gene and its relationship to MTHFR enzyme activity, homocysteine, and cardiovascular disease risk, J Mol Med-Jmm 79 (2001) $522-528$.

24. B.A. Maron, A. Loscalzo, The Treatment of Hyperhomocysteinemia, Annu Rev Med 60 (2009) 39-54.

25. F. Herrmann, A. Gross, D. Zhou, H.A. Kestler, M. Kuhl, A Boolean Model of the Cardiac Gene Regulatory Network Determining First and Second Heart Field Identity, Plos One 7 (2012).

26. J.I.E. Hoffman, S. Kaplan, The incidence of congenital heart disease, J Am Coll Cardiol 39 (2002) 1890-1900. Pii S07351097(02)01886-7. 